\title{
Weight Assessment of Some Accessory Digestive Organs in the Adult African Giant Pouched Rat (Cricetomys Gambianus, Waterhouse-1840)
}

NZALAK", J. O., IBE, C. S., DAUDA, S. E. and SULEIMAN, H. M.

Department of Veterinary Anatomy, Ahmadu Bello University, Zaria, Nigeria *Correspondence: E-mail: o.james42@yahoo.com. Tel.: +2348035973420

\section{INTRODUCTION}

The African giant pouched rat is a wild rodent of the Muridae family that is predominant in Africa, south of Sahara (Rosevear, 1969). They are adaptable to several environmental conditions including lowlands and highlands (Bigalke, 1964). The rats are widely distributed in all parts of Nigeria, where they are consumed as 'bushmeat'. The smoked carcasses of the rodent are often seen in village markets. Attempts have been made to breed and rear the rats in captivity, for food (Ajayi, 1975). For effective breeding and domestication, there is a need to quantify the digestive organs of the adult African giant pouched rat. This will give an insight into the efficiency of their digestive system, the knowledge of which may be employed in the breeding programmes, or as animal models for feed formulation and nutrient trials. This study was initiated based on the paucity of literature on the gross morphometry of accessory digestive organs in the adult African giant pouched rat. The study was aimed at determining the weights of the liver, gall bladder and pancreas in adult African giant pouched rats in both absolute and relative terms, and to compare the results with what has been reported in other rodents.

KEY WORDS: Liver, Gall bladder, Pancreas, Weight, African giant pouched rat

\section{MATERIALS AND METHODS}

Thirty eight adult African giant pouched rats, of both sexes, were captured alive in and around Samaru village in Zaria, Kaduna state, using locally made metal cage traps. They were transported to the Gross Anatomy Laboratory, Department of Veterinary Anatomy, Ahmadu Bello University Zaria. The animals were kept in standard laboratory cages and acclimatized for 2 weeks. During this period, the animals were given access to drinking water, raw sweet potatoes and groundnuts, ad libitum.

The rats were sedated using chloroform as the inhalation anaesthetic. The body weight of each rat was obtained with a digital electronic balance (Citizen Scales (1) PVT Ltd., sensitivity: $0.01 \mathrm{~g}$ ). The rats were then euthanized by an overdose of inhalation chloroform. With the animals in a supine position, laparotomy, through a mid-ventral abdominal incision, exposed the abdominal viscera. The location of the liver, gall bladder and pancreas and their relationship with other abdominal viscera were observed in situ. The organs were then exteriorized and their weights obtained using a sensitive electronic balance (Mettler balance P 1210, Mettler instrument AG. Switzerland; sensitivity: $0.001 \mathrm{~g}$ ).

\section{RESULTS AND DISCUSSION}

Table I shows a summary of the mean weights of the liver, gall bladder and pancreas obtained from the 38 adult African giant pouched rats.

\section{The liver}

The liver of the African giant pouched rat was found to be dark brown in colour, smooth and glossy in appearance, situated in the abdominal cavity, in contact with the stomach, duodenum, transverse colon, jejunum, spleen and right kidney. The colour of the liver in the present study was not very far from that of the Wistar rat as reported by Olds and Olds (1979). They stated that the liver of the Wistar rat is mahogany-brown in colour.

The liver of the African giant pouched rat, in the present study, had a mean weight of $22.91 \mathrm{~g}$. This constituted $2.1 \%$ of the mean body weight (Table I). This is also equivalent to one-fiftieth (1/50th) of the body weight. According to Caster 
et al. (1956), the liver of the laboratory rat constitutes $4.15 \%$ of the body weight. This implies that, in relative terms, the laboratory rat has a heavier liver than the African giant pouched rat.

\section{The Gall Bladder}

A gall bladder was observed in the African giant pouched rat. Rudolf and Stromberg (1976) and Olds and Olds (1979) reported that the Wistar rat lacks a gall bladder. The gall bladder in the African giant pouched rat was a sac-like organ on the visceral surface of the liver at the point where the median lobe of the liver was divided into 2 sub-lobes. The mean weight of the gall bladder was $0.34 \mathrm{~g}$. This constituted $0.03 \%$ of the body weight (Table I).

Table I: Morphometric Indices of the Liver, Gall bladder and Pancreas in the African giant Pouched Rat $(n=38)$

\begin{tabular}{llllc}
\hline Parameter & Minimum & Maximum & Mean \pm SEM & $\begin{array}{c}\text { Relative } \\
\text { weight(\%) }\end{array}$ \\
\hline Body weight $(\mathrm{g})$ & 800 & 1400 & $1077 \pm 68.28$ & 100 \\
Weight of Liver $(\mathrm{g})$ & 18.9 & 28.2 & $22.91 \pm 1.06$ & 2.1 \\
Weight of gall bladder $(\mathrm{g})$ & 0.2 & 0.5 & $0.34 \pm 0.03$ & 0.03 \\
Weight of pancreas $(\mathrm{g})$ & 0.6 & 1.4 & $0.97 \pm 0.07$ & 0.09 \\
\hline
\end{tabular}

\section{The Pancreas}

The pancreas of the African giant pouched rat was pale red in colour, soft, pliable and fragile. The pancreas lay on the characteristic U-shape of the duodenum. It branched and spread along the omentum between the stomach and the spleen.
The mean weight of the pancreas was $0.97 \mathrm{~g}$. This constituted $0.09 \%$ of the body weight (Table I).

\section{CONCLUSION}

The present study provided information on the weights of the liver, gall bladder and pancreas in adult African giant pouched rats. The base-line data will be valuable in further pharmacological and nutritional investigations involving these accessory digestive organs in the African giant pouched rat.

\section{REFERENCES}

AJAYI, S. S. (1975): Observations on the biology, domestication and reproductive performance of the African giant rat (Cricetomys gambianus, Waterhouse) in Nigeria. Mammalia, 39: 343364.

BIGALKE, R. C. (1964): Can Africa Produce New Domestic Animals? New Scientist, 374: 141146.

CASTER, W. O., PONCELET, J., SIMON, A. B. and ARMSTRONG, W. D. (1956): Tissue weights of the rat 1 . Normal values determined by dissection and chemical methods. Proc. Soc. Exp. Biol. Med. 91: 122-126.

OLDS, R. J. and OLDS, J. R. (1979): A Colour Atlas of the Rat. Dissection Guide. Wolfe Medical Publications Ltd., London, pp. 72-80.

ROSEVEAR, W. R. (1969): The Rodents of West Africa. Eyre and Spottiswoode, London.

RUDOLF, H. and STROMBERG, M. V. (1976): Anatomy of the Laboratory Rat. The Williams and Wilkins Company, pp. 50-54. 